\title{
APPLICATION OF THE H-MODE, A DESIGN AND INTERACTION CONCEPT FOR HIGHLY AUTOMATED VEHICLES, TO AIRCRAFT
}

\author{
Kenneth H. Goodrich, NASA Langley Research Center, Hampton VA \\ Frank O. Flemisch, DLR-Institute of Transportation Systems, Braunschweig Germany \\ Paul C. Schutte, NASA Langley Research Center, Hampton VA \\ Ralph A. Williams, Analytical Mechanics Associates, Hampton VA
}

\begin{abstract}
Driven by increased safety, efficiency, and airspace capacity, automation is playing an increasing role in aircraft operations. As aircraft become increasingly able to autonomously respond to a range of situations with performance surpassing human operators, we are compelled to look for new methods that help us understand their use and guide their design using new forms of automation and interaction. We propose a novel design metaphor to aid the conceptualization, design, and operation of highly-automated aircraft. Design metaphors transfer meaning from common experiences to less familiar applications or functions. A notable example is the "Desktop metaphor" for manipulating files on a computer.

This paper describes a metaphor for highly automated vehicles known as the $H$-metaphor and a specific embodiment of the metaphor known as the $H$-mode as applied to aircraft. The fundamentals of the H-metaphor are reviewed followed by an overview of an exploratory usability study investigating human-automation interaction issues for a simple $\mathrm{H}$-mode implementation. The envisioned application of the $\mathrm{H}$-mode concept to aircraft is then described as are two planned evaluations.
\end{abstract}

\section{Introduction}

Highly automated vehicles, motivated by improved performance, efficiency, and safety, are becoming commonplace in many forms of transportation. As used here, the term "highly automated" refers to hardware/software systems that can perform a majority of the control functions traditionally performed by a human operator. At the beginning of the 21st century, the automation of state of the art aircraft can control all its actions from just after take-off to touchdown and rollout during routine operations.

A large body of experience with highly automated airplanes highlights several fundamental challenges that limit current system effectiveness [1-4]. Briefly summarized, these challenges include heavy reliance on memorized action sequences, workload spikes caused by reformulation of operational tasks into subtasks understood by the automation, stupefying workload lulls, complacency, skill attrition, inflexible automation support, and sudden, unexpected changes in automation behavior. Furthermore, unless the basic form of human-machine interaction is improved, the adverse effects of these challenges are likely to be more pronounced as potentially less trained and supervised operators (e.g., single-pilots or remote operators versus a multi-pilot, commercial flight crew) use highly automated vehicles.

The current state of the art is typified by the flight management and control systems on contemporary transport aircraft (Figure 1). The crew can control the vehicle using three primary

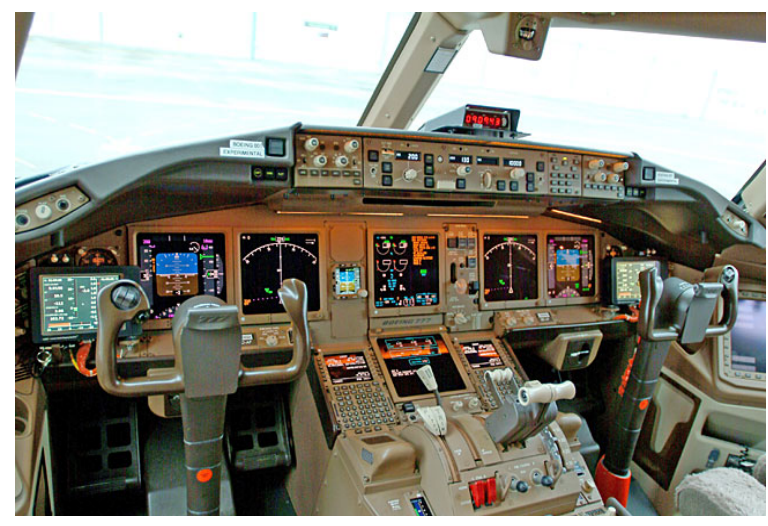

Figure 1. State of the art flight deck 
control automation levels corresponding, in general, to manual control, parameter control via an autopilot/auto-throttle system, or fully coupled operation through a flight management system (FMS). The interfaces for the three levels of control automation are designed as independent systems, resulting in three sets of interfaces (i.e., manual control inceptors, mode-control panel, and the FMS-Control Display Unit) and system characteristics that the operators must be proficient with, choose, and manage in any given situation. Control of the lateral path, vertical path and speed may be simultaneously spread across all three interfaces. This situation is complicated by the many sub-modes within the primary automation levels. These sub-modes are often associated with subtle but important changes in system behavior (e.g., whether or not stall protection is provided) increasing the likelihood of hazardous operator confusion. At the highest automation level (i.e., fully coupled operation via the FMS), commands are typically entered in the form of abstract, alphanumeric identifiers rather than a spatially encoded form (e.g., physically designating features on a map or in the external scene). This translation from one form to another increases the likelihood of accidents such as the crash of American Airlines flight 965 near Cali, Columbia [5] In this event, the crew mistakenly entered a waypoint unrelated to their intended route of flight. Having to look up and enter alpha-numeric identifiers can also create significant workload, particularly in complex, terminal area airspace where workload is often already high.

When utilizing highly automated modes of operation, the crew is effectively separated from the physical conduct of the flight. While they still have critical responsibilities such as monitoring for incipient conflicts or hazards, there is no direct feedback if these functions are not performed. Without regular involvement in the control of the vehicle or feedback if more supervisory tasks go unperformed, it is possible for crews to fall below a safe level of situation awareness, reducing their ability to mitigate emerging hazards or respond in time-critical situations. In addition, with no required physical interaction, functionally incapacitated crews may go undetected until the situation has already deteriorated to an extreme degree. An example of such an occurrence is the loss of the Learjet carrying Payne Stewart [6].

In addition to these conventional, crew controlled functions, safety and security research activities are currently developing concepts in which the automation may autonomously deviate from the crew's instructions in the presence of a significant conflict or hazard [7]. If such automation initiated actions occur only during rare, uncertain, time-critical, and potentially hazardous circumstances, crews are likely to have limited proficiency in predicting and verifying the correctness of its operation. This lack of familiarity increases the likelihood that a crew inappropriately disables or overrides the automation or conversely, delays a needed intervention.

Furthermore, traditional automation interfaces and associated input parameters (e.g., discrete parameter entry via knobs or buttons and alphanumeric identifiers via a keyboard or comparable input devices such as voice recognition) are illsuited to the management of spatially referenced, autonomous behaviors in dynamic situations. The inability to efficiently communicate simple but situationally referenced actions may force reversion to unaided manual control in many high workload situations. While eliminating the possibility of human intervention or limiting it severely (e.g., a wave-off button) may be seen as solutions to these concerns, for complex systems operating in uncertain environments, this approach is not without numerous and as yet unsolved issues $[8,9]$. The development of artificial systems with humanlike "common sense" and creative problem solving skills in real-world situations remains a "grand goal" as described by leading researchers such as Minsky [10]. From a practical perspective, systems that do not support flexible human interventions are likely to remain more expensive and less robust than systems that do for some time to come.

How then should we apply the "lessons learned" from late 20th century cockpit automation to the design of future aircraft? How do we balance between exploiting increasingly powerful technologies and retaining authority, with clear roles between humans and automation? Is there a new way to conceptualize a highly-automated vehicle and its operation; perhaps in the form of a metaphor? Design metaphors transfer meaning 
from common experiences to less familiar applications or functions. A ubiquitous example is the desktop metaphor that has been successfully applied to many personal computer operating systems for manipulating electronic documents. Not all aspects of the original need be copied as a metaphor typically has plasticity such that it can, and should be, shaped and adapted as necessary to support understanding. If the original reference is sufficiently intact and familiar to the user, a metaphor facilitates easy understanding and operation of the new application. Designers and engineers usually have to expend a great deal of effort in developing and communicating their intent not only to users, but also to others involved in the development, operation, and training processes. This communication can be improved by using a 'seed crystal' such as a suitable metaphor.

What could be an appropriate metaphor for automated vehicles? Let's leave technology for a moment and imagine a situation in everyday life:

\section{The H-Metaphor:}

Imagine you are riding your bicycle through a park with trees and people. You are late for an appointment so you're in a hurry. You're trying to avoid hitting anything and also get to your

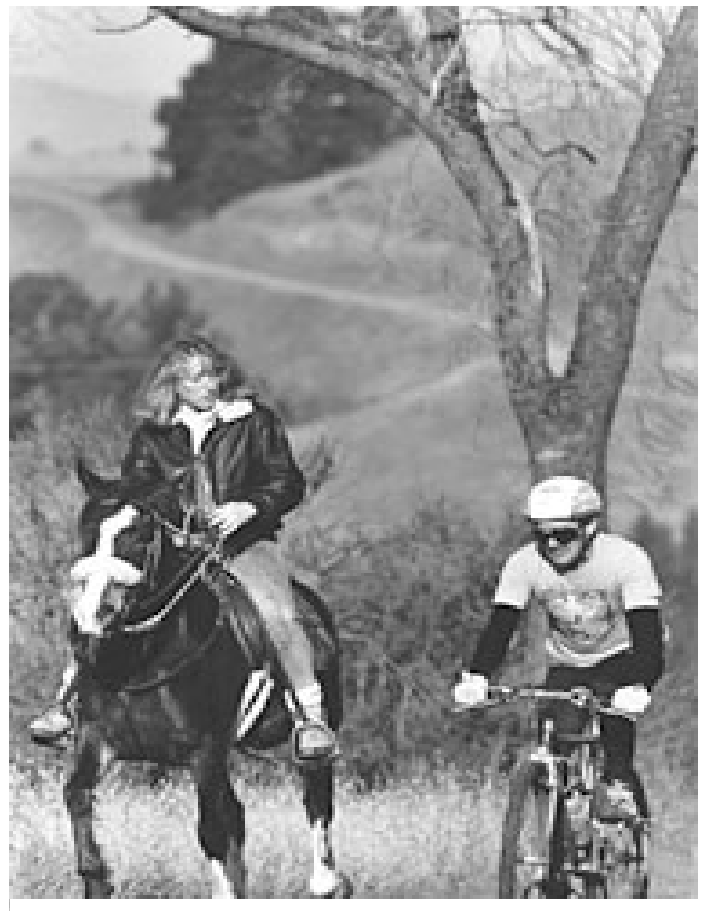

Figure 2. Horse as an autonomous vehicle archetype appointment on time, but you're not very familiar with this park and you need to keep referring to your map. The problem is that it is very difficult to steer your bike and read a map at the same time, so you are forced to stop. As the time for your appointment draws nearer you keep thinking to yourself - Is there a way to move through an environment with obstacles without having to stop every time you need to do something else? How about a Horse?! Riding a horse (Figure 2), you would be able to read your map and be confident that you would not hit any trees or run into people because horses instinctively avoid obstacles. Using Haptic (manipulative touch, a combination of tactile and kinesthetic cues) feedback through the seat of your pants and your reins, you are constantly aware of what your horse is doing, even while focusing your attention elsewhere. If the horse is unsure about where to go, it will slow down, and seek a new obstacle free path while trying to get you back into the loop. The horse might also be aware of how engaged you are and adjust its behavior. If a dangerous situation suddenly pops up, it will try to react before it is too late. You can let your horse choose its path without being out-ofthe-loop or you can take it on tight rein to reassert a more direct command.

Now apply this image to an aircraft. Imagine flying through complex airspace with other traffic, weather, and tall obstructions while comfortably attending to other tasks like navigation, communication, or visually searching the surrounding terrain. Through the physical feedback from your haptic interface, you are constantly aware of what your airplane is doing. If your airplane senses a conflict, it will take appropriate action and you can feel where it's leading. You can let your aircraft choose its path without being completely out-of-the-loop, or you can reassert more direct control by taking a tighter grip on the stick. The airplane would be aware of how engaged you are in the control task and adjust its behavior accordingly. An extreme example would if the pilot became incapacitated. In this event, the airplane would declare an emergency and divert to an appropriate airport. An H-Metaphor inspired aircraft like this could make flying significantly safer and more productive. The metaphor is perhaps best suited to applications such as single-pilot and Unmanned Aircraft System (UAS) operations. These 
applications have a strong incentive for adopting advanced technologies and are not well served by simple extrapolations from transport aircraft-like interfaces.

\section{Overview of the H-Metaphor}

The H-metaphor as detailed in Flemisch, et al. [11] and summarized here, structures the relationship between operator and an intelligent vehicle as a partnership between two dissimilar, but cooperative agents sharing control of a vehicle (e.g., Figure 3). This partnership is motivated by the emergence of highly automated vehicles capable of autonomously performing a wide variety of tasks with great precision and vigilance combined with

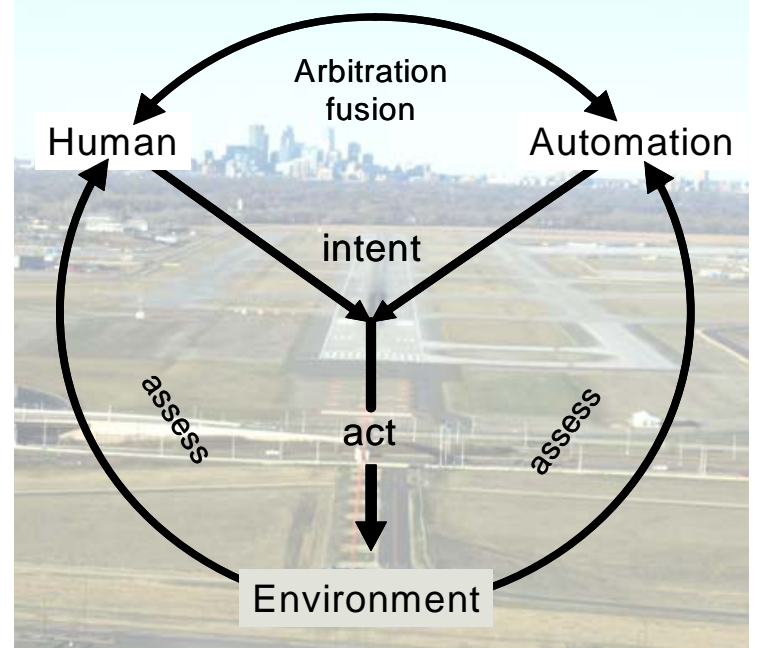

Figure 3. Shared control between cooperative Agents

the recognition that their specialized intelligence will still be relatively brittle when faced with realworld complexities. For example, the ability to determine whether a sensed object is a benign empty bag or a hazardous chunk of concrete could be important in a variety of situations but is far from feasible in any general sense. The $\mathrm{H}$ metaphor addresses this situation by relying on the general intelligence of a human operator. In the partnership, the human strategizes, corroborates, and backs up actions of the automation in the accomplishment of operations that are beyond the vehicle's reliable, independent cognition. The human is aided in this role by the vehicle's awareness of the routine situation elements; ability to perform immediate trajectory control tasks; the intelligence of its interaction; and its ability to independently monitor and maintain immediate operational safety. The goals of this coupling are improved overall system safety, performance, and flexibility beyond what could be accomplished with conventional automation or manual control while significantly reducing the cost and complexity of the needed automation and operator training. Furthermore, the metaphor provides a path for the incremental introduction of increasingly capable automation technologies and may be a necessary evolutionary step toward, and in many cases an alternative to, autonomous vehicles that routinely operate in complex, safety-critical environments without human backup.

The metaphor provides guidance on the human-vehicle interaction, the vehicle as an autonomous agent, and multi-vehicle interaction [11]. A horse and rider system interacts through multi-modal communication underpinned by a strong, bi-directional haptic component. Human haptic senses (e.g., proprioception and cutaneous touch) are unique in that they both sense and effect the external environment. Furthermore, as a vector quantity (i.e., force) they provide a natural means of communicating spatial concepts such as the direction of an intended or desired maneuver. These properties enable robust and rapid, bidirectional communication and negotiation between horse and rider. For example, even in the case of a horse cart without direct bodily contact, the reins form a bi-directional link with the horse able to transmit information to the driver as well as receive it. According to the German National Equestrian Federation, turns are performed by yielding the outside rein with a twist of the hand(s), not pulling the inside one (Figure 4). There should always be a "soft, steady, elastic connection" between the driver's hand and horse's mouth, where "the horse
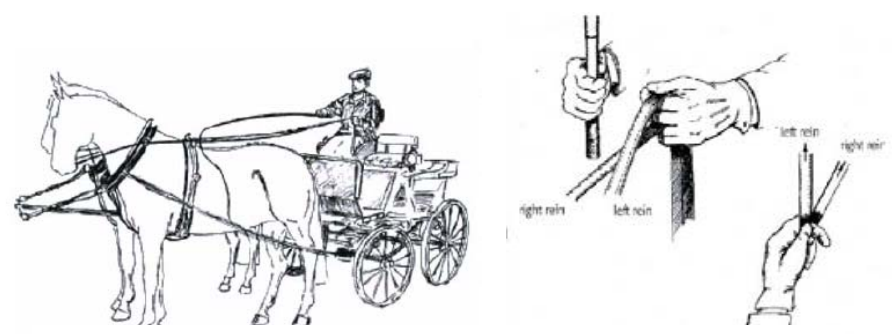

Figure 4. Hands and reins position for elastic connection [12] 
seeks the contact and the driver provides it" [12]. If the horse wants to change direction, perhaps to avoid a rut, it leads with a turn of its head. The rider feels a change in rein tension and can accept or reject the action by relaxing or increasing the tension. In addition, discrete communication elements such as an agitated jerk (possibly accompanied with an auditory snort and visual tail flick) are often combined with such continuous signals. Furthermore, from a lack of any tension the horse can infer that the driver is relatively disengaged and adjust its expectations accordingly.

Efficient bi-directional communication results in a type of shared control not present in conventional automation. Conventional automation tends to be 'all or nothing' where either the human or the machine has control. In comparison, the rider of a horse can hold the locus of control while the horse remains "in-the-loop" performing innerloop functions and monitoring for hazards (i.e., tight reins operation). Alternatively, the rider can also explicitly or implicitly designate a clear task or behavior of some duration for the horse to perform and allow the horse to have the locus of control (e.g., loose reins). The rider, even in loose reins, remains in-the-loop and can flexibly control important aspects of the behavior such as what position to maintain relative to another, running animal (e.g., calf roping ) or how to avoid a hazard (e.g., left, right or over).

It should be recognized that the H-metaphor applies to the near-term or tactical actions of the vehicle. Horses, outside of TV and movies, do not worry about what is hours ahead relative to the goals of the rider. As part of a complete system concept, a separate, but complementary planning sub-system is envisioned to support longer term, mission planning and monitoring requirements (e.g., Schutte and Goodrich [13]). The maximum look-ahead of an $\mathrm{H}$-inspired system is at the discretion of the designers, but in keeping with the metaphor, the human-automation interaction in particular, would nominally project only as far as needed to support the current action and possible transitions while accounting for local hazards. While it may seem counterintuitive to limit the ability to pre-program sequences of action as far into the future as technologically feasible, the intent of the limitation is two fold. The first is to ensure that the operator has regular, but relaxed, physical involvement throughout a mission, thus aiding maintenance of situation awareness. The second motivating factor is optimizing the interaction for robust communication of near-term, possibly time and safety critical goals and actions. This near-term focus serves to minimize miscommunication and/or protracted exchanges by keeping the language set and range of situationally applicable behaviors small and with a minimum of abstraction.

\section{The H-mode, a Realization}

The metaphor provides a broad vision as to how an H-inspired vehicle should interact and behave. Achieving this vision requires better understanding the fundamental human-automation interaction issues, applying and advancing the underlying technologies for autonomous systems, and integrating this knowledge into practical designs for specific operational applications such as ground vehicles, aircraft and spacecraft. Ideally, this work should result in specific, yet malleable automation design and user interaction guidelines that could be applied across a wide range of “intelligent" vehicles. By facilitating positive transfer of training, knowledge, and experience between operational domains and reducing the potential for confusion, we could expect significant improvements in safety, operational effectiveness and training productivity for both users and developers.

The H-mode, a practical realization of the metaphor using commercially available haptic interface hardware (e.g., an active, force-feedback side-stick) and with applicability across operational domains, is currently being developed by NASA and the German Aerospace Lab, DLR. Early steps in this process include an exploratory study conducted first at NASA and latter extended at the DLR. As described by Flemisch, Goodrich, and Conway [14], this study investigated the general user acceptance and expectations of the metaphor and developed an initial, generic "language" for the interaction between a simple automated vehicle and a human operator. A small but broadly representative group of prototypical users participated in a structured interview to explore their "naïve" reaction to the metaphor and expectations for, and acceptance of, an intelligent 
$\mathrm{H}$-inspired vehicle. The results of these interviews (the questionnaire and responses are reproduced in ref. 14), indicate overall acceptance of the metaphor and that the general issues and capabilities of such vehicles are intuitively understood.

The interaction investigation consisted of a "structured exploration" to develop and evaluate a preliminary interaction language between a simple automated vehicle simulation and a human operator using an active side-stick for the following generic 1-dimensional control tasks:

- Starting and Stopping the vehicle

- Maintaining a desired spacing interval behind a lead vehicle. As shown in figure 5, deviations from the desired interval were indicated by triangular zones of increasing risk and rectangular zones of significant danger. The subjects and " $\mathrm{H}$ " did not necessarily have the same zones, requiring a haptic negotiation to find the best compromise.

- Transitioning between a more automated and a less automated mode of operation (Tight Rein/Loose Rein)

The operator performed the last two tasks in parallel with a secondary task to mimic the divided attention likely in actual operations.

A goal of the exploration was to match the expectations of users (e.g., "What behavior would you expect of this artifact in that situation?") with the technical potential of the design space and the ideas of the designer. One of the tools used to achieve this match was a "theater" system or "Wizard of Oz" technique, based on the concept of Salber and Coutaz [14]. A confederate "behind the curtain" simulated parts of the automation via a second side-stick that was electronically linked to

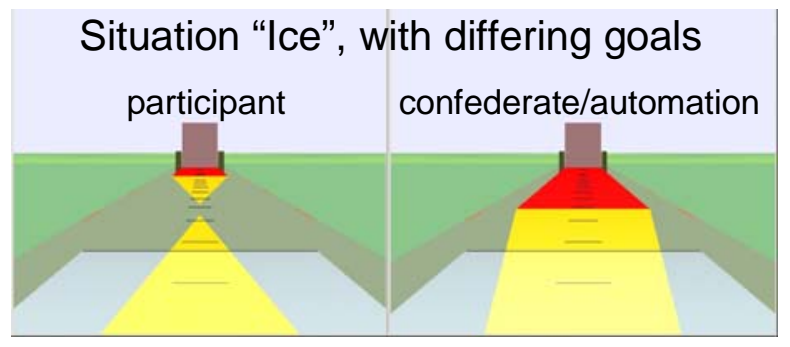

Figure 5. Distance task from ref. 14

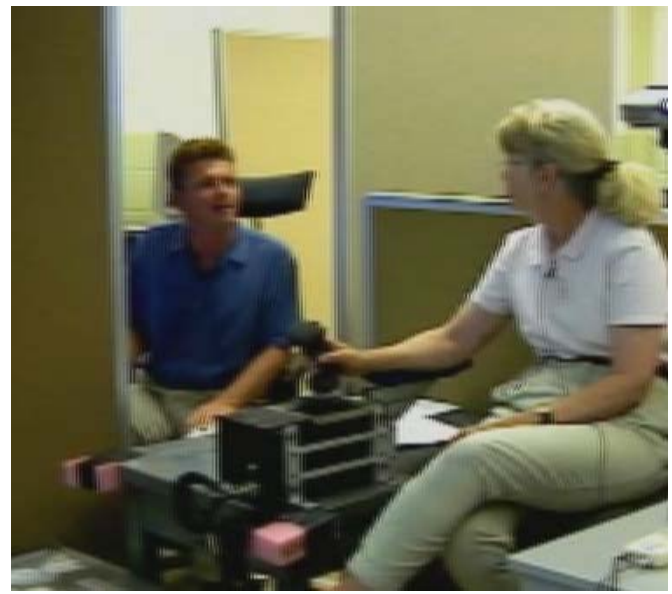

Figure 6. Theater system with curtain open

the subject's stick. The technique allowed flexible exploration of users expectations and possible design variations prior to interactions with a rapid prototype implemented in technology. While in the original technique described in ref. 15, the participant does not know about the confederate, here the participant knew about it from the very beginning. The "curtain" was open in the training phases (Figure 6) and closed during the usability assessments.

The exploration went through the following stages:

- Pre-Design of potential interaction patterns by a core design team in the theater.

- Usability assessment and refinement of the predesign with other team members.

- Rapid prototyping implementation of selected features of the pre-design.

- Structured interview with prototypical users.

- Laboratory based assessment with prototypical users.

o Collection of expectations directly in the theater system

o "Naïve runs" (users interact with Pre-design without any training)

o Teaching of the pre-design and comparison to expectations

o Usability assessment of pre-design with the theater system

o Usability assessment of pre-design with the rapid prototypes 


\section{o De-Briefing}

- Harmonization of the pre-design with the expectations

While a detailed description of the results goes beyond the scope of this paper, a first draft of interaction consists of the following haptic communication cornerstones:

- Vibrations: A specific scheme of vibrations that combines information about danger and degree of automation ("Alive"-vibration), combined with

- Pushing: A specific scheme of pushing back the stick from danger, combined with

- Signaling: Discrete interaction “double-ticks” for signaling the desire for discrete distance or speed changes, combined with

- Transitioning: A specific scheme of fluidly transitioning between a higher level of automation (Loose Rein) and a lower level of automation (Tight Rein)

o Tight Rein $\rightarrow$ Loose Rein by first leading the $\mathrm{H}$-vehicle into the desired maneuver and then (Variant 1) relaxing the hand on the stick or (Variant 2) a discrete "yes"-signal or (Variant $3)$ a combination of 1 and 2.

o Loose Rein $\rightarrow$ Tight Rein by taking a firm grip, if necessary combined with a discrete "no"-signal.

- Fine Tuning: A specific scheme of fine tuning reference values the automation in Loose Rein

- Arbitration: Specific schemes of prioritizing between the will of the user and of the automation

While a couple of additional design ideas surfaced and a few glitches were found during the usability assessment, overall the pre-design matched most of the expectations of the evaluation subjects and the prototypes were, for this state of development, quite intuitive.

\section{Toward a Comprehensive Aircraft Implementation}

DLR and NASA are currently investigating more comprehensive applications of the H-mode to ground and air vehicles, respectively. The current status of the ground vehicle application is summarized in Flemisch, Schieben, and Schindler [16] and the aviation application is described here.

\section{Technical Perspective}

Figure 7 illustrates the conceptual system architecture. Major system elements include the multi-modal user interface including an active or haptic side-stick and speed-command lever (not shown); inner-loop control augmentation; real-time sensors and/or data link system; and the H-inspired automation (HIA).

Within the user interface, the active stick and speed-command lever provide a common means by which both the operator and HIA issue commands to the inner-loop. The haptic interaction allows each agent to sense the others involvement (e.g., applied force) and infer an associated intent. Visual displays (i.e., a primary flight and tactical map displays) provide graphical representations of the external environment overlaid by basic flight parameters and the HIA's interpretation of this information. Since the inner-loop automation provides full-time stability and control augmentation, presentation of basic flight parameters is primarily for awareness rather than control and can be significantly simpler than current formats. The displayed environmental information includes physical elements such as terrain, obstructions, weather, and traffic and virtual elements such as airspace boundaries, published fixes and procedures. The route from a mission planning system, if present, is also included. Elements rendered on the displays are also known to the HIA. HIA specific symbology allows the pilot to quickly ascertain its status, motivation, center of attention, projected trajectory, and how it would respond to potential directives. Finally, although not currently implemented in a prototype, auditory interface features will be used to compliment the haptic and visual elements. In keeping with the H-metaphor, these communications can be bi-directional but limited in content and complexity (e.g., not complex sentences or abstractions).

The output of the stick provides the reference signal acted on by the inner-loop control element, essentially a fly-by-wire autopilot providing flightpath oriented, control-wheel steering and envelope protection (e.g., [17-20]). Considering the horse 


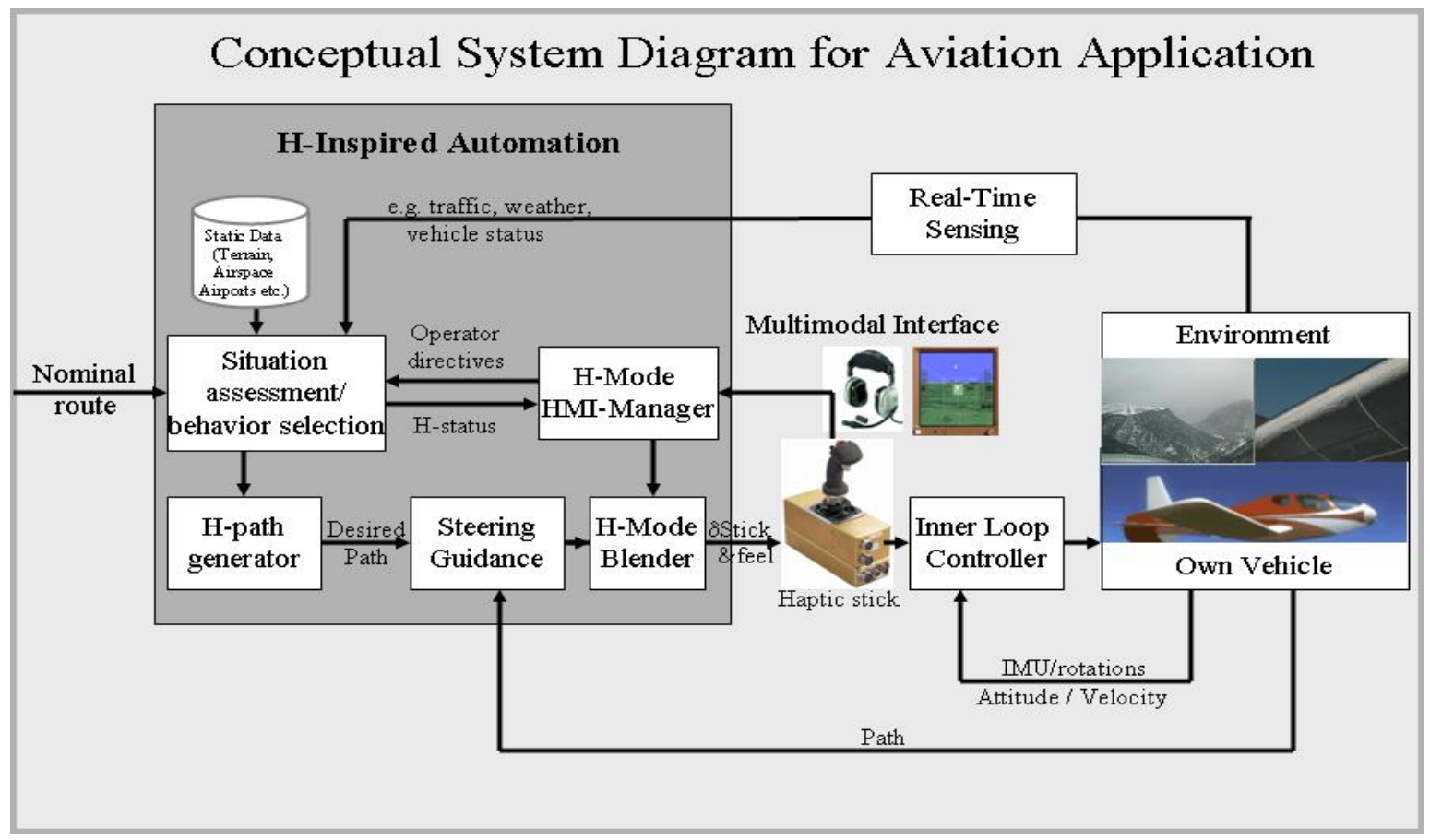

Figure 7. H-mode based flight control system

metaphor, the inner-loop can be likened to the hind brain, spinal cord, and voluntary neuromuscular system. The inner-loop simplifies the pilot-vehicle interaction into an easily managed set of elemental actions. This simplification is accomplished by delegating management of a number of highfrequency, non-linear, but largely deterministic control processes to the inner-loop. This aggregation also reduces the workload and training of the operator and simplifies the design and understanding of the outer-loop automation elements. Obviously, the effects of possible failure modes and other "non-normals" need to be considered on overall system behavior. The current state of the art is rapidly enabling retention of augmented response characteristics in the presence of component failures, albeit with some performance degradation, rather than reversion to a completely different set of dynamics (e.g., a direct electric link mode). Optional, advanced functions within the inner-loop include upset recovery and adaptive processes compensating for vehicle changes caused by airframe damage or other effects (e.g., airframe icing) such as described by Burken, et al [21]. For maximum capability, these functions should communicate operating envelope changes to other system elements (i.e., HAI, interaction manager) so that they can appropriately update their internal models.

The sensor and data link capabilities allow the vehicle to obtain real-time information about the local environment, functioning as the external senses of the metaphor. These sensors could include conventional instruments such as satellite based navigation (e.g., GPS), weather radar, etc, as well has emerging technologies like multi-spectral machine vision for navigation and hazard avoidance and digital data link based state and intent sharing between vehicles and air traffic control. One of the benefits of the $\mathrm{H}$-mode concept is that new sensor capabilities can be readily integrated into its framework rather than being added in a stand alone fashion with "integration" largely left to the pilot as is often done today.

Within the HIA, relevant static information stored in databases is analogous to long term memory. This static data provides the HIA with knowledge of nonphysical elements such as airways, static physical features such as terrain, and databases characterizing its own capabilities. Within the situation assessment function, real-time 
and static data are analyzed, features identified, and an integrated representation of the current situation constructed. The situation assessment also includes relevant inputs from the operator. The important aspects of the perceived situation are communicated back to the operator via the interaction manager. When operating in loose reins mode, the HIA creates and maintains a desired trajectory which is followed by a guidance subsystem. In tight reins mode, the current aircraft implementation does not generate a reference trajectory and no maneuvering command is sent to the stick unless an imminent conflict is perceived. Alternatively, the DLR is investigating the soft haptic presentation of a desired trajectory even during tight reins operation. This presentation may assist the operator and increase their awareness of potential future actions by the HIA and be particularly useful in situations where the scope of safe tight reins maneuvering is limited.

Various means of conflict avoidance are under investigation at both organizations. Currently, simple reactive behaviors such as potential-field guidance [22] appear to be an intuitive, robust and predictable means of performing obstacle avoidance. In addition, superposition of individual guidance commands (e.g., path following + avoidance) aided by simple heuristics, is being investigated as a means of effectively reacting to simultaneous influences.

\section{Pilot's Perspective}

The H-mode system integrates control of the vehicle's full range of control automation into a single consistent interface system and natural set of interaction skills. The pilot uses the same basic control and display devices for augmented manual control through complex behaviors such as takeoffs, landings, coupled procedures, and automated conflict/hazard avoidance.

The H-mode emphasizes two basic modes of operation corresponding to tight and loose reins. In the tight reins mode, the pilot holds the locus of immediate control while in loose reins the aircraft autoflight system has a relatively high degree of autonomy in satisfying longer-term directives. Transitions between tight and loose are managed by an "arbitration function" in the interaction manager. Other modes of operation supporting more extensive autonomous actions are possible design extensions but are not discussed here as they are perhaps best viewed outside the context of the metaphor.

In tight reins, the pilot commands the nearterm actions of the vehicle through the inner-loop control and does not communicate any sustained intent to the HIA. In this mode, the HIA provides no or limited aid unless it perceives an emerging conflict or hazard. In the event of a perceived conflict, the HIA brings the situation to the pilot's attention via the visual and haptic interface elements. If the conflict severity exceeds a defined threshold, the automation sends a force input, effectively a maneuver command, to the stick. If unopposed, this input safely redirects the vehicle. The pilot can also perform their own deconfliction maneuver or, if for some reason they need to continue toward the conflict, apply an appropriate counter-force. The degree and duration of the automation's resistance can be situationlly tailored by the arbitration function. For example, once the pilot has demonstrated an unambiguous and informed intent to continue toward a hazard, it may be advisable for the automation to reduce or cease its opposition. Such situations might arise due to erroneous or incomplete local situation awareness and/or knowledge of the mission objective by the HIA. Limiting the strength and/or duration of opposition provides a mechanizm through which the pilot retains authority.

In loose reins, the HIA performs a well defined task or behavior. This behavior may be initiated through explicit direction from the pilot or implicit in the local situation (e.g., flare before touchdown). Flying published procedures or a preplanned route are examples of actions normally performed in loose rein. Several behaviors may be combined such as route following while maintaining spacing from other traffic. Other loose rein behaviors include precise, high-bandwidth tasks such as takeoff and flare and landing.

In loose reins, the pilot is relieved from continuously generating instantaneous commands but remains actively involved in managing and transitioning between behaviors. By lightly touching the haptic interface elements, the pilot can feel the HIA's instantaneous commands, providing a non-visual means of maintaining awareness. This 
awareness is aided by discrete haptic signals indicating the general situation status and imminent events such as reaching an intersection or planned trajectory transition (e.g., top of descent).

The pilot also uses the haptic interface to modify key parameters within a behavior and manage transitions between behaviors. Within a behavior, pilot applied forces can be fed back to the HIA and used to update its active reference targets in an operationally intuitive manner. For example, in a following task, pressure on the speed command lever is fed back to adjust the separation interval. Similarly, in a landing behavior, light stick forces can be used to adjust the aim point used in the landing guidance. The HIA's receptiveness to these updates is situationally tailored such that only safe and appropriate actions are supported in loose reins. The pilot's awareness of this receptiveness is supported via multi-modal cues including tailoring of the basic feel characteristics of the haptic interface (e.g., a heavier feel as limits are approached).

At major transition or decision points, the HIA can be configured to react only after the pilot has made a positive control input indicating they are aware of the situation. The pilot typically makes this input by manually initiating the transition. This interaction helps prevent the detachment and loss of situation awareness that can occur with conventional automation and encourages the pilot to verify the appropriateness of major transitions. If the pilot fails to initiate an expected transition, the aircraft provides increasingly salient cues. If the pilot still does not respond, the automation shifts toward a more conservative (i.e., safer) posture or action. At decision height, for example, the pilot is expected to make an input confirming that it is safe to proceed and transition from the approach to the landing behavior. If this input is not sensed, the aircraft initiates a missed approach. The pilot can nominally alter or reject an expected transition by making a light control input consistent with an alternate loose-reins action or by making a firm input, triggering a transition to tight reins.

If the HIA perceives an emerging conflict during loose reins operations, it brings the situation to the operator's attention. If the HIA is capable of generating a safe, straightforward deconfliction maneuver, it will be offered as an option to the pilot. Like a normal decision point, the pilot can initiate the action with a simple, positive control input while remaining in loose reins. If not satisfied with any of the loose reins resolution actions, the pilot has the option of transitioning into tight reins. If the pilot does not respond, or if there is insufficient time to allow reaction, the HIA autonomously shifts toward a conservative posture.

Transitions between loose and tight reins are managed by an arbitration function in the humanmachine interaction manager. The interaction manager interprets the pilot's inputs in the context of the HIA's perceived situation. This interpretation is aided by a small set of discrete elements allowing simple, but direct communication, similar to the interaction commonly seen between a human and a well trained animal. These elements include signals meaning 'yes', 'no', 'NO', 'I am unsure', and 'I want your attention'. The interaction manager typically encodes these multi-modally including a haptic component. Current research is exploring a variety of generation methods from the pilot's side, including haptic signals and dedicated buttons.

In general, if the pilot sustains a firm input while in loose reins, a transition into tight reins occurs. If, while operating in tight reins, the pilot maintains the vehicle in a specified relationship relative to certain external elements, the automation offers or initiates the appropriate loose reins behavior. Figure 8, for example, shows the pilot directing the automation to enter a published holding pattern. First, the pilot maneuvers the airplane so that the flight path marker (FPM) symbol is stabilized on a visualization of the fix associated with the hold. Rendered as a transparent "pole", the visualization allows the pilot to graphically specify the altitude of the procedure. The vertical extent of the pole indicates the range of appropriate altitudes and the projection of the FPM on the pole, rounded to the nearest appropriate increment, specifies the desired altitude. As the pilot stabilizes on the pole, the HIA highlights the procedure, indicating that it can transition to loose reins. The pilot completes the transition via a "yes" signal and a magenta line provides a visual indication of the trajectory guiding the automation. Simultaneously, the basic feel characteristics of the stick are changed to indicate the vehicle has the 
1. Pilot maneuvering toward fix in tight reins

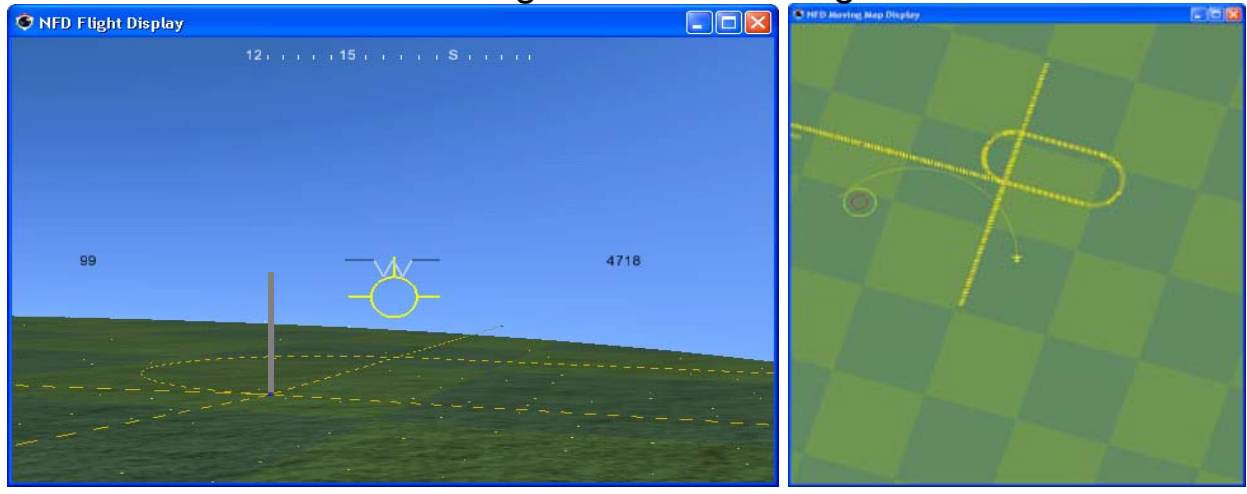

2. HIA "sees" fix, offers appropriate loose-reins behavior

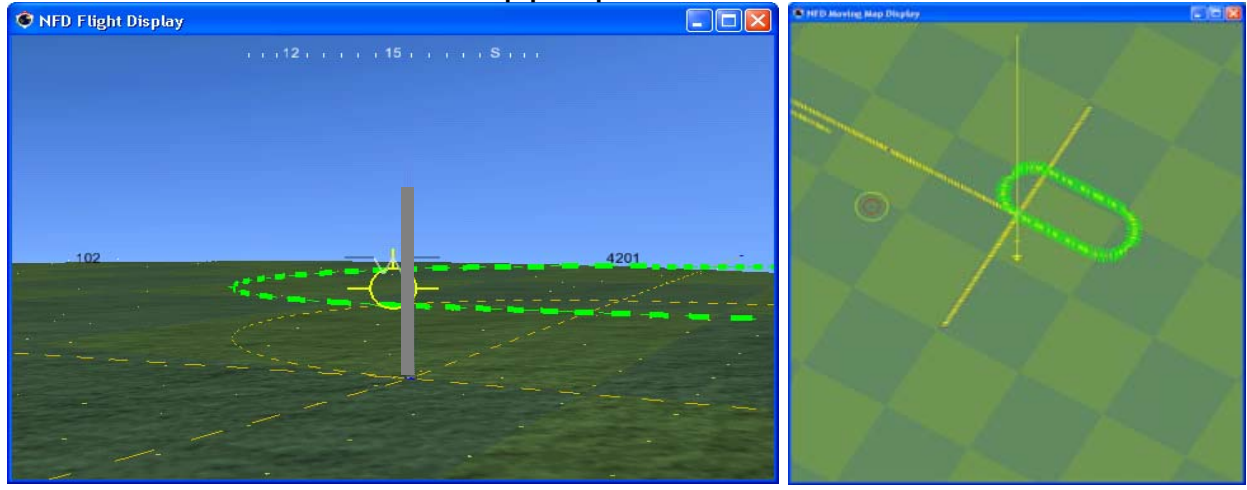

3. Pilot accepts, transition to loose-reins complete

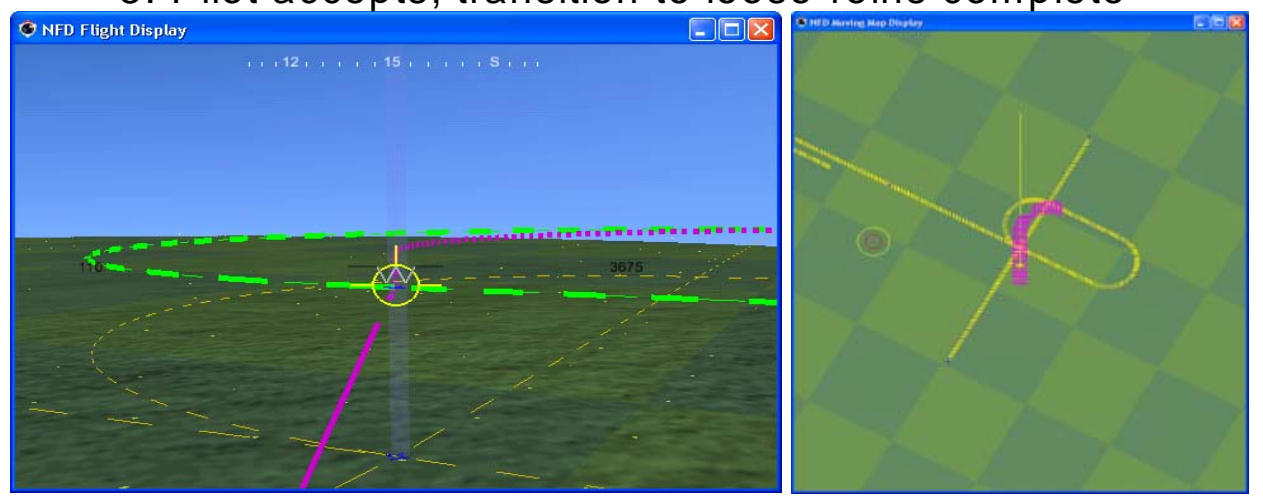

Figure 8. Transition from tight to loose riens

locus of control. In some situations, several loose reins behaviors may be applicable and additional discrete communication is used to select or clarify the desired action. Situations in which the HIA requests a transition from loose to tight will be encountered as it becomes unsure of the situation. Depending on the situation, the operator may or may not have the option of declining the transition.

\section{Planned Evaluations}

The functionality described above has been developed at NASA Langley through several simulation prototypes, building up from the simple $1 \mathrm{D}$ vehicle and environment used in the exploratory usability study, through planar operations in 2D, and currently in a light-aircraft simulation and 
environment representative of the terminal area around a typical general aviation airport. So far, these prototypes have been implemented in a laboratory simulation environment on PC class computers. Presently, the functionality is being ported to the NASA Langley's Visual/Motion Simulator. A study investigating the effects of aircraft motion, turbulence, and extraneous cockpit vibrations on the usability of the $\mathrm{H}$-mode concept in general and on specific types of haptic interaction is to be conducted in this simulator within the next 6 months. A second study is planned in the laboratory simulation in this same timeframe. This study will investigate the interactions between control automation level (i.e., augmented manual control; conventional, fully coupled automation; and an implementation of the H-mode), task performance, pilot workload, and situation awareness. While each automation level may perform best relative to a specific task element or performance measure, the objective is to assess whether the H-mode provides improved overall performance across the full range of task and measures.

If these simulation studies provide positive results, it would be desirable to proceed to initial flight implementations. Such an implementation would be fairly straightforward on existing systems research aircraft such as the Bonanza described by Duerksen [18]. Implementations on unmanned/ remotely supervised aircraft would also be of high value and interest.

\section{Summary and Discussion}

The H-mode defines a specific, yet malleable human-machine interface design, associated human-machine interactions, and system architecture that is applicable to a wide range of vehicles and operational environments. By integrating management of the full range of control automation functions into a single consistent interface system and natural set of interaction skills, many of the fundamental human-machine interaction problems exhibited by conventional, highly automated vehicles may be reduced or eliminated. Also, by facilitating positive transfer of training, knowledge, and experience between operational domains and reducing the potential for confusion, we expect significant improvements in safety, effectiveness and training productivity for both users and developers. Additionally, unlike conventional automation implementations, the breadth and malleability of H-mode concept allows integration of future technologies without changing the fundamental system design and associated operator skills. Further, the concept provides a pathway by which emerging automation technologies supporting increasingly complex and safety-critical behaviors can be introduced and operationally evaluated while retaining effective human oversight.

Much research and development remains before these claims may be realized, but the potential combined benefits of increased safety, ease of use, and performance make it a worthwhile task. NASA and the DLR are investigating comprehensive aerospace and ground vehicle applications respectively. At NASA, a simulated prototype for aircraft has been developed in a laboratory environment. This simulation is currently being ported to a motion-base simulator where the effects of motion on the concepts overall usability and design space will be evaluated. A second experiment exploring the interaction between automation level, workload, task performance, and situation awareness is also planned for the near future. Positive results from these experiments should establish the basic feasibility and impact of the H-mode concept in aircraft operations, and combined with the work at the DLR, will guide the future development of this promising concept.

\section{References}

[1] Billings, Charles, 1997, Aviation automation: The Search for a Human-Centered Approach. Mahwah, NJ: Lawrence Erlbaum Associates.

[2] FAA Human Factors Team, 1996, “The Interfaces Between Flightcrews and Modern Flight Deck Systems”, Washington D. C., FAA

[3] Wiener Earl, 1998, “Cockpit Automation”, In E. L. Wiener \& D. C. Nagel (Eds.), Human Factors In Aviation, San Diego, CA: Academic Press, pp. 433461

[4] Sherry, Lance; Peter, Polson; Michael, Feary, 2001, "Designing User-Interfaces for the Cockpit: Five Common Design Errors and how to Avoid 
Them”, Society of Automotive Engineers, 02WAC98, Warrendale, PA

[5] NTSB, 1996, Washington, D. C., National Transportation Safety Board, www.ntsb.gov/ntsb/ GenPDF .asp?id=DCA96RA020\&rpt $=$ fa

[6] NTSB, 1999, Washington, D. C., National Transportation Safety Board, www.ntsb.gov/publictn/2000/AAB0001.pdf

[7] Andy Pasztor, 2006, “Airbus Planes to Use Computers In Crash-Avoidance Maneuvers”, Wall Street Journal, May 27 2006, New York, NY, Dow Jones and Company

[8] Steering Committee for the Decadal Survey of Civil Aeronautics, 2006, "Decadal Survey of Civil Aeronautics: Foundation for the Future", Washington, D. C., The National Academies Press

[9] Williams, Kevin, 2004, “A Summary of Unmanned Aircraft Accident/Incident Data: Human Factors Implications”, Oklahoma City, OK, Civil Aerospace Medical Institute, FAA

[10] Minsky, Marvin; Push Singh; Aaron Sloman, 2004, "The St. Thomas Common Sense

Symposium: Designing Architectures for HumanLevel Intelligence”, AI Magazine, summer 2004, Menlo Park, CA, AAAI Press

[11] Flemisch, Frank, C. Adams, S. Conway, K. Goodrich, M. Palmer, P. Schutte, 2003, “The Hmetaphor as a guideline for vehicle automation and interaction”, Technical Memorandum No. NASA/TM-2003-212672, Hampton, VA, LaRC

[12] German National Equestrian Federation, 2002, The Principles of Driving, Buckingham, UK, Kenilworth Press.

[13] Schutte, Paul, Ken Goodrich, et al 2006, “The Naturalistic Flight Deck System: An Integrated System Concept for Improved Single-Pilot Operations”, Draft TM, Hampton, VA, LaRC

[14] Flemisch, Frank; Ken Goodrich, Shelia Conway, 2005, "ITS At the crossroads of manually controlled and automated transport: The $\mathrm{H}$ -

Metaphor and its first applications (progress update 2005)”, Hannover Germany, Presented at The 5th European Congress and Exhibition on Intelligent Transport Systems and Services HIT
[15] Salber, Daniel, Joëlle Coutaz, 1993, “Applying the Wizard of $\mathrm{Oz}$ Technique to the Study of Multimodal Systems”; INTERCHI 93 Conference, New York, NY, pp 95-96.

[16] Flemisch, Frank, J. Kelsch, A. Schieben, J. Schindler, 2006, "Stücke des Puzzles hochautomatisiertes Fahren: H-Metapher und HMode“, 4. Workshop Fahrerassistenzsysteme; Löwenstein, Germany

[17] Lambregts, Anthony, 2005, "Fundamentals of Fly-By-Wire Augmented Manual Control”, Society of Automotive Engineers, 05WAC-62, Warrendale, PA

[18] Duerksen, Noel, 2003, “Advanced Flight Controls and Pilot Displays for General Aviation”, AIAA 2003-2647. 2003 AIAA/ICAS International Air and Space Symposium and Exposition, Dayton $\mathrm{OH}$

[19] Liu, Dahai, Ken Goodrich, Bob Peak, 2006 "Study of Synthetic Vision Systems (SVS) and Velocity-vector Based Command Augmentation System (V-CAS) on Pilot Performance”, Institute of Industrial Engineers Annual Conference and Exposition 2006, Orlando FL

[20] Borst C., M. Mulder, M. M. van Paassen, J. A. Mulder, 2006 "Path-Oriented Control/Display Augmentation for Perspective Flight-Path Displays”, Journal of Guidance, Control, and Dynamics, Vol. 29, Reston, VA, AIAA

[21] Burken, John, Peggy Williams-Hayes, John Kaneshige, Susan, Stachowiak, 2006, "Reconfigurable Control with Neural Network Augmentation for a Modified F-15 Aircraft", NASA TM-2006-213678, DFRC, Edwards, CA

[22] Arkin, Ronald 1998, Behavior-Based Robotics, Cambridge, Massachusetts, MIT Press

\section{$25^{\text {th }}$ Digital Avionics Systems Conference October 15, 2006}

